\title{
Endoscopic Transsphenoidal Salvage Surgery for Symptomatic Residual Cystic Craniopharyngioma after Radiotherapy
}

\author{
Enzo Emanuelli ${ }^{1}$ Giuliana Frasson ${ }^{1}$ Diego Cazzador ${ }^{1}$ Daniele Borsetto ${ }^{1}$ Luca Denaro ${ }^{2}$
}

${ }^{1}$ Department of Neurosciences, Otorhinolaryngology Section,

Address for correspondence Enzo Emanuelli, MD, Department of University of Padua, Padua, Italy

Neurosciences, Otorhinolaryngology Section, University of Padua, Via

${ }^{2}$ Department of Neurosciences, Neurosurgery Section, University of Giustiniani 2, 35128, Padua, Italy (e-mail: enzoemanuelli@libero.it).

Padua, Padua, Italy

J Neurol Surg B 2018;79(suppl S3):S256-S258.

\begin{abstract}
\section{Keywords}

- cystic craniopharyngioma

- childhood brain tumor

- endoscopic transsphenoidal surgery

- anterior skull base

Objectives Ideal treatment of craniopharyngiomas is still controversial. Radiotherapy (RT) is considered effective for recurrences or after subtotal tumor removal (STR). About 40 to $50 \%$ of patients may experience tumor cyst expansion soon after RT; in these cases, the role of salvage surgery is debated.

Design Operative video.

Setting Tertiary care center.

Participants An 11-year-old boy diagnosed with persistent craniopharyngioma. In 2015, the patient underwent right frontotemporal craniotomy for STR at another center, complicated by panhypopituitarism. Two years later, fractionated 54-Gy RT was performed on growing residual tumor. After 3 months, he was admitted to our hospital due to persistent malaise, vomiting, pulsating headache, and epistaxis. Ophthalmologic evaluation evidenced left homonymous hemianopsia.

Results A contrast-enhanced magnetic resonance imaging (MRI) showed a 27-mm cystic component enlarging from the cranial end of the persistent craniopharyngioma lesion, extending into the third ventricle. Biventricular hydrocephalus and brain midline shift to the right were present. Compared with the early post-RT MRI, the cystic component of the tumor demonstrated growth. The patient underwent external ventricular drainage placement for emergent treatment of hydrocephalus and endoscopic transsphenoidal surgery. After cystic content drainage, the lesion was completely removed with its capsule. A "gasket seal" technique was performed for skull base reconstruction, with autologous fascia lata, septal bone, and mucoperiosteum from inferior turbinate. Histologic examination confirmed the craniopharyngioma diagnosis. Postoperative MRI showed resolution of the hydrocephalus and complete tumor removal.
\end{abstract}

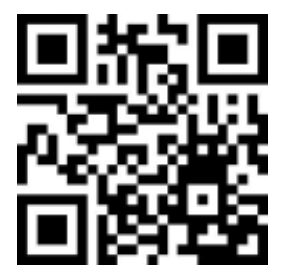

www.thieme.com/skullbasevideos

www.thieme.com/jnlsbvideos

received

October 15, 2017

accepted

December 12, 2017

published online

February 8, 2018
DOI https://doi.org/

10.1055/s-0038-1636504.

ISSN 2193-6331. (c) 2018 Georg Thieme Verlag KG

Stuttgart · New York
License terms

(c) (i) $\ominus$ (5) 
Conclusion Although shrinkage of cystic components of craniopharyngioma residuals may occur within 5 to 6 months after RT, salvage surgery is indicated in symptomatic patients.

The link to the video can be found at https://youtu.be/4x6Qe76bf60.

Conflict of Interest

None.

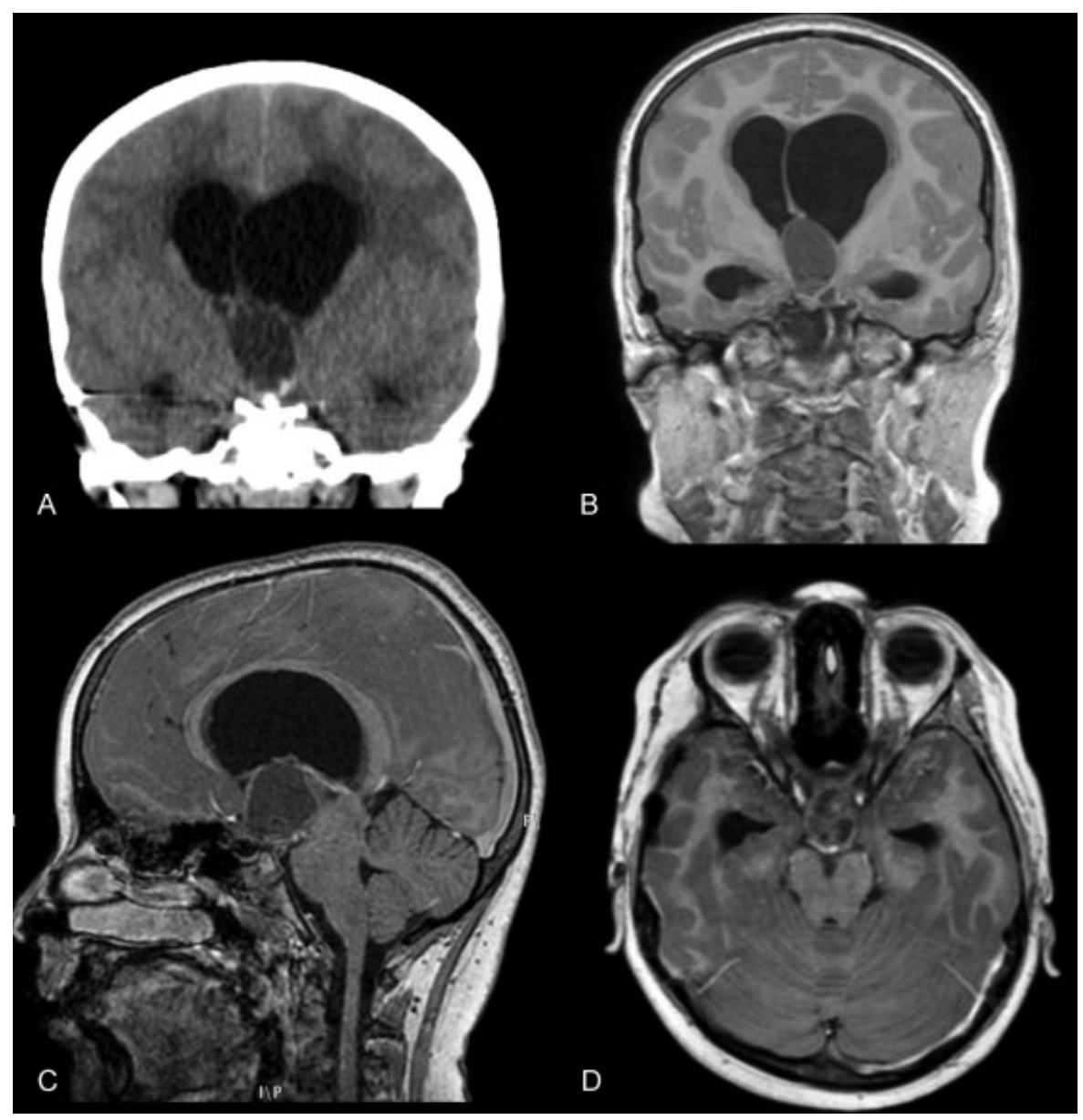

Fig. 1 Coronal CT scan (A) and coronal contrast-enhanced T1-weighted MRI scan (B) showing the cystic component of craniopharyngioma occupying the third ventricle, with biventricular hydrocephalus. Sagittal contrast-enhanced T1-weighted MRI scan (C) evidenced a solid component in the sellar region, displacing anteriorly the optic chiasm (D). CT, computed tomography; MRI, magnetic resonance imaging. 


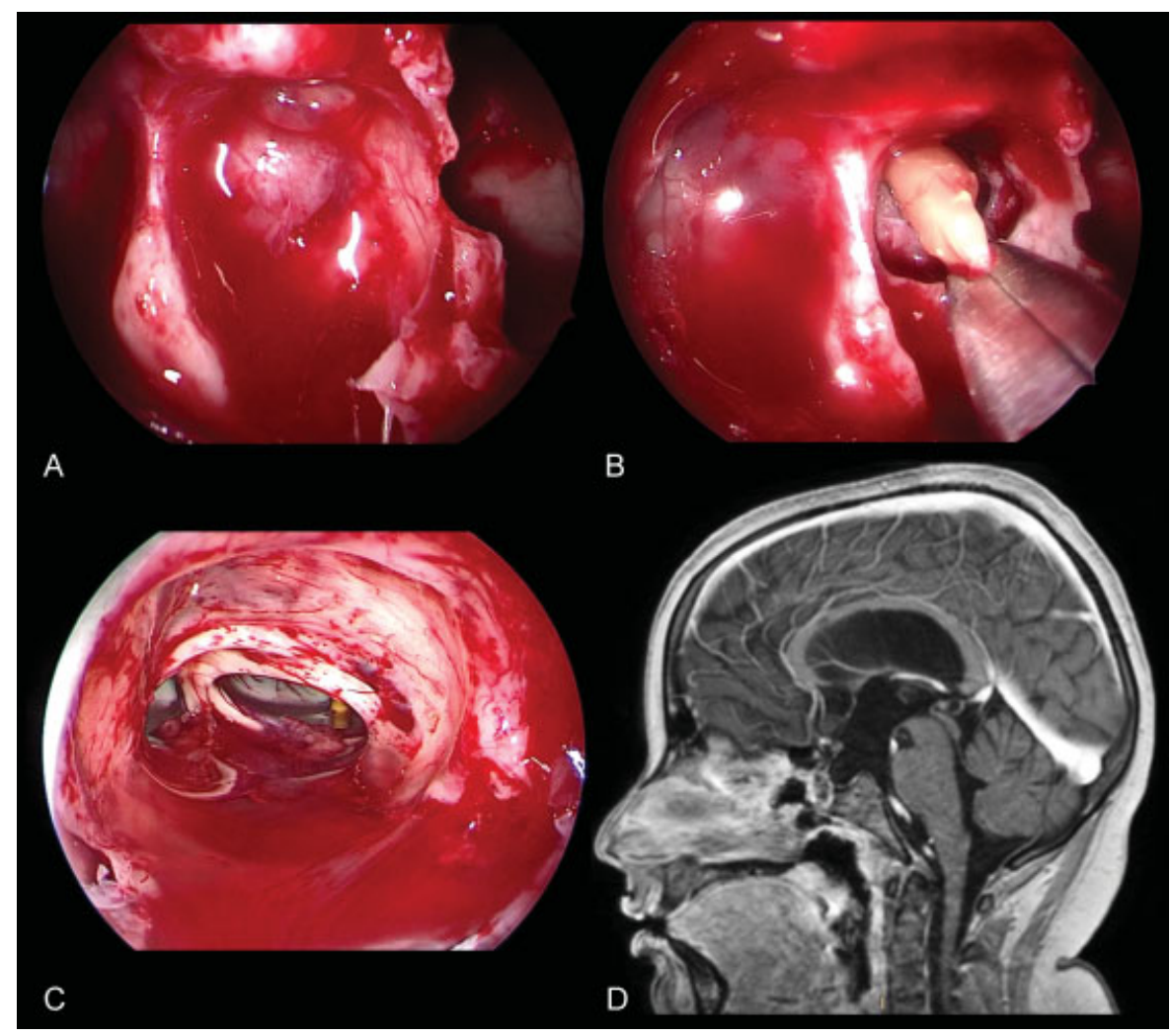

Fig. 2 Intraoperative view with 0-degree endoscope showing the dehiscence of the anterior sellar wall (A) and the removal of the tumor capsule (B) with an endoscopic transsphenoidal approach. Intraoperative view with 45-degree endoscope after tumor removal through the third ventricle, evidencing the placement of the external ventricular shunt in the left lateral ventricule (C). Postoperative sagittal contrast-enhanced T1-weighted MRI (D). MRI, magnetic resonance imaging. 